\title{
THE PLASMA OSCILLATIONS IN A TWO-DIMENSIONAL ELECTRON-HOLE LIQUID
}

\author{
S. A. Moskalenko ${ }^{1}$, M. A. Liberman ${ }^{2}$, E. V. Dumanov ${ }^{1}$, A. G. $\operatorname{Stefan}^{1}$, M. I. Shmiglyuk ${ }^{1}$ \\ ${ }^{1}$ Institute of Applied Physics of the Academy of Sciences of Moldova, \\ Academic St. 5, Chisinau, MD2028, Republic of Moldova \\ ${ }^{2}$ Department of Physics, Uppsala University, Box 530, SE-751 21, Uppsala, Sweden
}

(Received September 21, 2007; received in final form July 18, 2008)

\begin{abstract}
The plasma oscillations of the two-dimensional electron-hole system in the presence of a strong perpendicular magnetic field are studied. Only the intra-Landau level excitations are taken into account when the electrons and holes are situated on their lowest Landau levels, the filling factor $v^{2}$ being less than 1 . The ground state of the two-dimensional e-h system is supposed to be the electron-hole liquid. The dispersion relations for the optical and acoustical plasmon modes were obtained. The influence of the supplementary in-plane electric field acting side by side with the strong perpendicular magnetic field is discussed.
\end{abstract}

Key words: plasma oscillations, electron-hole liquid, optical plasmons, acoustical plasmons.

PACS number(s): 71.35.Lk, 71.35.Ee

\section{INTRODUCTION}

The plasma oscillations in the three-dimensional (3D) bulk crystals as well as in the two-dimensional (2D) layers in the system created by one-component electron gas are characterized by the squared frequencies, which obey a common formula

$$
\omega_{p}^{2}(q)=2 N_{e} T_{q} V_{q}
$$

where $N_{e}$ is the total number of electrons, $T_{q}$ is their kinetic energy $T_{q}=\frac{\hbar^{2} q^{2}}{2 m}$, and $V_{q}$ is the Fourier transform of the electron-electron Coulomb interaction.

In the case of $3 \mathrm{D}$ systems we have $V_{q}=\frac{4 \pi e^{2}}{\varepsilon_{0} V q^{2}}$, where $\mathrm{V}$ is the volume of the crystal and is the dielectric constant of the medium. It leads to the well known results [1]

$$
\omega_{p}^{2}(q)=\frac{4 \pi e^{2} n_{e}}{\varepsilon_{0} m}, \quad n_{e}=\frac{N_{e}}{V}
$$

For the 2D structures we have [2-4]

$$
\omega_{p}^{2}(q)=\frac{2 \pi e^{2} n_{S} q}{\varepsilon_{0} m}, \quad V_{q}=\frac{2 \pi e^{2}}{\varepsilon_{0} S q^{2}}, \quad n_{S}=\frac{N_{e}}{S} .
$$

Here $S$ is the surface area of the 2D-layer. Das Sarma and Madhukar [2] considered the two-component twodimensional electron gas (2DEG). Two oscillations with density fluctuation operators $\hat{\rho}_{1}(q)$ and $\hat{\rho}_{2}(q)$ combine each other forming the optical and acoustical plasmon oscillations with the frequencies $\omega_{\mathrm{OP}}(q) \sim \sqrt{q} ; \omega_{\mathrm{AP}}(q) \sim q$;

The plasmon oscillations in one-component system on the monolayer in a strong perpendicular magnetic field were studied by Girvin, MacDonald and Platzman [5], who proposed the magnetoroton theory of collective excitations in the conditions of the fractional quantum Hall effect. Some properties of electron-hole system in a strong perpendicular magnetic field were discussed in [6-9].

Below we will study similar questions in the case of a $2 \mathrm{D}$ e-h system when the filling factor $v^{2}$ of the lowest Landau levels(LLLs) is smaller than $1\left(v^{2}<1\right)$ and the ground state of the system is supposed to be the electron-hole liquid (EHL).

The paper is organized as follows. In the first chapter we will study the optical and acoustical plasma oscillations due to intra-LLLs excitations. In the second chapter the influence of a supplementary lateral electric field on the plasma frequencies will be discussed.

\section{OPTICAL AND ACOUSTICAL PLASMA MODES}

The Hamiltonian of the Coulomb interaction of electrons and holes in the frame of the lowest Landau levels is $[10]$

$$
H=\frac{1}{2} \sum_{\mathbf{Q}} W_{\mathbf{Q}}\left[\hat{\rho}(\mathbf{Q}) \hat{\rho}(-\mathbf{Q})-\hat{N}_{e}-\hat{N}_{h}\right] .
$$

Here $W_{\mathbf{Q}}$ is the Fourier transform of the Coulomb interaction. $\hat{N}_{e}$ and $\hat{N}_{h}$ are the operators of the full numbers of electrons and holes.

The density fluctuation operators for electrons $\hat{\rho}_{e}(\mathbf{Q})$ and for holes $\hat{\rho}_{h}(\mathbf{Q})$ as well as their linear combinations $\hat{\rho}(\mathbf{Q})$ and $\hat{D}(\mathbf{Q})$ are represented:

$$
\begin{aligned}
\hat{\rho}_{e}(\mathbf{Q}) & =\sum_{t} e^{i Q_{y} t l^{2}} a_{t-\frac{Q_{x}}{2}}^{\dagger} a_{t+\frac{Q_{x}}{2}} \\
\hat{\rho}_{h}(\mathbf{Q}) & =\sum_{t} e^{i Q_{y} t l^{2}} b_{t+\frac{Q_{x}}{2}}^{\dagger} a_{t-\frac{Q_{x}}{2}} \\
\hat{\rho}(\mathbf{Q}) & =\hat{\rho}_{e}(\mathbf{Q})-\hat{\rho}_{h}(-\mathbf{Q}) ; \\
\hat{D}(\mathbf{Q}) & =\hat{\rho}_{e}(\mathbf{Q})+\hat{\rho}_{h}(-\mathbf{Q}) ;
\end{aligned}
$$


where $l=\sqrt{\hbar c / e H}$ is the magnetic length.

These operators obey the following commutation relations:

$$
\begin{aligned}
& {[\hat{\rho}(\mathbf{Q}), \hat{\rho}(\mathbf{P})]=-2 i \sin \left(\frac{[\mathbf{P} \times \mathbf{Q}]_{z} l^{2}}{2}\right) \hat{\rho}(\mathbf{P}+\mathbf{Q})} \\
& {[\hat{D}(\mathbf{Q}), \hat{D}(\mathbf{P})]=-2 i \sin \left(\frac{[\mathbf{P} \times \mathbf{Q}]_{z} l^{2}}{2}\right) \hat{\rho}(\mathbf{P}+\mathbf{Q})} \\
& {[\hat{\rho}(\mathbf{Q}), \hat{D}(\mathbf{P})]=-2 i \sin \left(\frac{[\mathbf{P} \times \mathbf{Q}]_{z} l^{2}}{2}\right) \hat{D}(\mathbf{P}+\mathbf{Q}) .}
\end{aligned}
$$

The motion equation for the operator is obtained using the commutation relations (6). It is [10]:

$$
\begin{aligned}
i \hbar \frac{d \hat{\rho}(\mathbf{P})}{d t} & =E(\mathbf{P}) \hat{\rho}(\mathbf{P})-2 i \sum_{\mathbf{Q}} W_{\mathbf{Q}} \sin \left(\frac{[\mathbf{P} \times \mathbf{Q}]_{z} l^{2}}{2}\right) \\
& \times \hat{\rho}(\mathbf{Q}) \hat{\rho}(\mathbf{P}-\mathbf{Q}) .
\end{aligned}
$$

Following the Zubarev method [11] Green's function $G_{1}(\mathbf{P}, \omega)=\left\langle\left\langle\hat{\rho}(\mathbf{P}) \mid \hat{\rho}^{\dagger}(\mathbf{P})\right\rangle\right\rangle_{\omega}$ was introduced. Its motion equation looks as follows

$$
\begin{aligned}
& (\hbar \omega+i \delta) G_{1}(\mathbf{P}, \omega)=C-2 i \sum_{\mathbf{Q}} W_{\mathbf{Q}} \sin \left(\frac{[\mathbf{P} \times \mathbf{Q}]_{z} l^{2}}{2}\right) \\
& \times\left\langle\left\langle[\hat{\rho}(\mathbf{P}-\mathbf{Q}) \hat{\rho}(\mathbf{Q})+\hat{\rho}(\mathbf{Q}) \hat{\rho}(\mathbf{P}-\mathbf{Q})] \mid \hat{\rho}^{\dagger}(\mathbf{P})\right\rangle\right\rangle_{\omega} .
\end{aligned}
$$

Writing new motion equation for the two operators Green's function $\left\langle\left\langle\hat{\rho}(\mathbf{Q}) \hat{\rho}(\mathbf{P}-\mathbf{Q}) \mid \hat{\rho}^{\dagger}(\mathbf{P})\right\rangle\right\rangle_{\omega}$ the following equations were obtained:

$$
\begin{aligned}
& G(\hat{P}, \omega)(\hbar \omega+i \delta)^{2}=C-\sum_{\mathbf{Q}} \sum_{\mathbf{R}} \sin \left(\frac{[\mathbf{P} \times \mathbf{Q}]_{z} l^{2}}{2}\right) \sin \left(\frac{[\mathbf{Q} \times \mathbf{R}]_{z} l^{2}}{2}\right) \\
& \times\left[\left\langle\left\langle\hat{\rho}(\mathbf{R}) \hat{\rho}(\mathbf{Q}-\mathbf{R}) \hat{\rho}(\mathbf{P}-\mathbf{Q}) \mid \hat{\rho}^{\dagger}(\mathbf{P})\right\rangle\right\rangle_{\omega}+\left\langle\left\langle\hat{\rho}(\mathbf{Q}-\mathbf{R}) \hat{\rho}(\mathbf{R}) \hat{\rho}(\mathbf{P}-\mathbf{Q}) \mid \hat{\rho}^{\dagger}(\mathbf{P})\right\rangle\right\rangle_{\omega}\right. \\
& \left.+\left\langle\left\langle\hat{\rho}(\mathbf{P}-\mathbf{Q}) \hat{\rho}(\mathbf{R}) \hat{\rho}(\mathbf{Q}-\mathbf{R}) \mid \hat{\rho}^{\dagger}(\mathbf{P})\right\rangle\right\rangle_{\omega}+\left\langle\left\langle\hat{\rho}(\mathbf{P}-\mathbf{Q}) \hat{\rho}(\mathbf{Q}-\mathbf{R}) \hat{\rho}(\mathbf{R}) \mid \hat{\rho}^{\dagger}(\mathbf{P})\right\rangle\right\rangle_{\omega}\right] \\
& -\sum_{\mathbf{Q}} \sum_{\mathbf{R}} \sin \left(\frac{[\mathbf{P} \times \mathbf{Q}]_{z} l^{2}}{2}\right) \sin \left(\frac{[(\mathbf{P}-\mathbf{Q}) \times \mathbf{R}]_{z} l^{2}}{2}\right)\left[\left\langle\left\langle\hat{\rho}(\mathbf{Q}) \hat{\rho}(\mathbf{R}) \hat{\rho}(\mathbf{P}-\mathbf{Q}-\mathbf{R}) \mid \hat{\rho}^{\dagger}(\mathbf{P})\right\rangle\right\rangle_{\omega}\right. \\
& \left.+\left\langle\left\langle\hat{\rho}(\mathbf{Q}) \hat{\rho}(\mathbf{P}-\mathbf{Q}-\mathbf{R}) \hat{\rho}(\mathbf{R}) \mid \hat{\rho}^{\dagger}(\mathbf{P})\right\rangle\right\rangle_{\omega}+\left\langle\left\langle\hat{\rho}(\mathbf{R}) \hat{\rho}(\mathbf{P}-\mathbf{Q}-\mathbf{R}) \hat{\rho}(\mathbf{Q}) \mid \hat{\rho}^{\dagger}(\mathbf{P})\right\rangle\right\rangle_{\omega}+\left\langle\left\langle\hat{\rho}(\mathbf{P}-\mathbf{Q}-\mathbf{R}) \hat{\rho}(\mathbf{R}) \hat{\rho}(\mathbf{Q}) \mid \hat{\rho}^{\dagger}(\mathbf{P})\right\rangle\right\rangle_{\omega}\right] .
\end{aligned}
$$

Acting in the same way we could receive the infinite chain of motion equations. This chain of motion equations can be truncated expressing the three operator Green's functions through the starting one operator Green's function multiplied by the average value of the type $\langle\hat{\rho}(\mathbf{Q}) \hat{\rho}(-\mathbf{Q})\rangle_{\omega}$ as follows:

$$
\begin{aligned}
&\left\langle\left\langle\hat{\rho}(\mathbf{P}-\mathbf{Q}-\mathbf{R}) \hat{\rho}(\mathbf{R}) \hat{\rho}(\mathbf{Q}) \mid d^{\dagger}(\mathbf{P})\right\rangle\right\rangle_{\omega} \approx G(\mathbf{P}, \omega)\left[\delta_{k r}(\mathbf{Q}, \mathbf{P})\langle\hat{\rho}(\mathbf{R}) \hat{\rho}(-\mathbf{R})\rangle+\left(\delta_{k r}(\mathbf{R},-\mathbf{Q})+\delta_{k r}(\mathbf{R}, \mathbf{P})\right)\langle\hat{\rho}(\mathbf{Q}) \hat{\rho}(-\mathbf{Q})\rangle\right] \\
&\left\langle\left\langle\hat{\rho}(\mathbf{P}-\mathbf{Q}) \hat{\rho}(\mathbf{R}) \hat{\rho}(\mathbf{Q}-\mathbf{R}) \mid d^{\dagger}(\mathbf{P})\right\rangle\right\rangle_{\omega} \approx G(\mathbf{P}, \omega)\left[\delta_{k r}(\mathbf{Q}, 0)\langle\hat{\rho}(\mathbf{R}) \hat{\rho}(-\mathbf{R})\rangle+\left(\delta_{k r}(\mathbf{R}, \mathbf{Q}-\mathbf{P})+\delta_{k r}(\mathbf{R}, \mathbf{P})\right)\right. \\
&\times\langle\hat{\rho}(\mathbf{P}-\mathbf{Q}) \hat{\rho}(\mathbf{Q}-\mathbf{P})\rangle] .
\end{aligned}
$$

The average value was calculated for the case of electron-hole liquid (EHL) with the filling factor $v^{2}$ of the lowest Landau levels (LLLs) for electrons and holes. In this approximation the dispersion relations $\omega_{\mathrm{AP}}(P)$ for acoustical plasmons and $\omega_{\mathrm{OP}}(P)$ for optical plasmons were obtained. They are

$$
\begin{aligned}
& \left(\hbar \omega_{\mathrm{AP}}(\mathbf{P})\right)^{2}=\sum_{\mathbf{Q}} W_{\mathbf{Q}}^{2} \times \sin ^{2}\left(\frac{[\mathbf{P} \times \mathbf{Q}]_{z} l^{2}}{2}\right)\langle\hat{\rho}(\mathbf{Q}) \hat{\rho}(-\mathbf{Q})\rangle ; \\
& \left(\hbar \omega_{\mathrm{OP}}(\mathbf{P})\right)^{2}=\sum_{\mathbf{Q}} W_{\mathbf{Q}}\left(W_{\mathbf{Q}}-W_{\mathbf{P}-\mathbf{Q}}\right) \times \sin ^{2}\left(\frac{[\mathbf{P} \times \mathbf{Q}]_{z} l^{2}}{2}\right)\langle\hat{\rho}(\mathbf{Q}) \hat{\rho}(-\mathbf{Q})\rangle .
\end{aligned}
$$

In the case of EHL the average value equals to

$$
\langle\hat{\rho}(\mathbf{Q}) \hat{\rho}(-\mathbf{Q})\rangle=2 N v^{2}\left(1-v^{2}\right) .
$$

The dispersion law for the acoustical plasmon has a linear dependence on the wave vector in the range of long wavelength and tends to constant value at great values of $P$. The dispersion relation for optical plasmons has a quadratic dependence at small values of $P$ and a similar behavior at great values of $P$ as in the case of acoustical plasmons. The difference between them is essential only in the range of intermediary values of $P$. 


\section{2D ELECTRON-HOLE SYSTEM IN A STRONG PERPENDICULAR MAGNETIC FIELD AND A LATERAL ELECTRIC FIELD}

The wave functions of $2 \mathrm{D}$ electrons and holes in a crossed magnetic and electric fields in Landau gauge have the forms [12]

$$
\begin{aligned}
& \psi_{p, n}^{i}(y)=\frac{e^{i p x}}{\sqrt{L_{x}}} \varphi_{n, p}^{i}(y) ; \\
& \varphi_{n=0, p}^{i}(y)=\frac{1}{\sqrt{l \sqrt{\pi}}} \exp \left[-\frac{\left(y-y_{p}^{i}\right)^{2}}{2 l^{2}}\right]
\end{aligned}
$$

where

$$
\begin{aligned}
& y_{p}^{i}=\frac{q_{i}}{e} l^{2}\left(-p+\frac{m_{i} V_{d}}{\hbar}\right) ; \quad i=e, h ; \quad q_{i}=\mp e \\
& E_{n, p}^{i}=-\frac{m_{i} V_{d}^{2}}{2}+\hbar V_{d} p+\hbar \omega_{c i}\left(n+\frac{1}{2}\right) ; \\
& V_{d}=c \frac{E}{H} .
\end{aligned}
$$

The operators of the density fluctuations $\hat{\rho}_{i}(\mathbf{Q})$ in the presence of lateral electric field are denoted as $\hat{\rho}_{i}^{E}(\mathbf{Q})$ and are determined by the formulas

$$
\begin{aligned}
& \hat{\rho}_{e}^{E}(\mathbf{Q})=e^{-i Q_{y} u_{e}} \hat{\rho}_{e}(\mathbf{Q}) \\
& \hat{\rho}_{h}^{E}(\mathbf{Q})=e^{-i Q_{y} u_{h}} \hat{\rho}_{h}(\mathbf{Q}) ; \\
& u_{i}=\frac{V_{d}}{\omega_{c i}} ; \omega_{c i}=\frac{e H}{m_{i} c} \\
& \hat{\rho}^{E}(\mathbf{Q})=\hat{\rho}_{e}^{E}(\mathbf{Q})-\hat{\rho}_{h}^{E}(-\mathbf{Q}) ; \\
& \hat{D}^{E}(\mathbf{Q})=\hat{\rho}_{e}^{E}(\mathbf{Q})+\hat{\rho}_{h}^{E}(-\mathbf{Q})
\end{aligned}
$$

They obey commutation relations

$$
\begin{aligned}
{\left[\hat{\rho}^{E}(\mathbf{Q}), \hat{\rho}^{E}(\mathbf{P})\right]=} & -2 i \sin \left(\frac{[\mathbf{P} \times \mathbf{Q}]_{z} l^{2}}{2}\right) \\
& \times \hat{\rho}^{E}(\mathbf{P}+\mathbf{Q}) ; \\
{\left[\hat{D}^{E}(\mathbf{Q}), \hat{D}^{E}(\mathbf{P})\right]=} & -2 i \sin \left(\frac{[\mathbf{P} \times \mathbf{Q}]_{z} l^{2}}{2}\right) \\
& \times \hat{\rho}^{E}(\mathbf{P}+\mathbf{Q}) ; \\
{\left[\hat{\rho}^{E}(\mathbf{Q}), \hat{D}^{E}(\mathbf{P})\right]=} & -2 i \sin \left(\frac{[\mathbf{P} \times \mathbf{Q}]_{z} l^{2}}{2}\right) \\
& \times \hat{D}^{E}(\mathbf{P}+\mathbf{Q}) .
\end{aligned}
$$

The Hamiltonian describing the 2D electrons and holes in the crossed magnetic and electric fields being situated in their lowest Landau levels has the from

$$
\begin{aligned}
& H^{E}=H_{0}+H_{\text {Coul }}^{E} \\
& H_{0}=\hbar V_{d} p\left(a_{p}^{\dagger} a_{p}+b_{p}^{\dagger} b_{p}\right) ; \\
& H_{\text {Coul }}^{E}=\frac{1}{2} \sum_{\mathbf{Q}} W_{\mathbf{Q}}\left[\hat{\rho}^{E}(\mathbf{Q}) \hat{\rho}^{E}(-\mathbf{Q})-\hat{N}_{e}-\hat{N}_{h}\right] .
\end{aligned}
$$

Considering the plasma excitations in their conditions can observe that the Hamiltonian leads to the previous self-energy parts, in which the frequency $\hbar \omega(P)$ is substituted by $\hbar \omega(P)-\hbar V_{d} P_{x}$.

\section{ACKNOWLEDGMENTS}

This research was supported by the common grant of the Russian Foundation for Fundamental Research and of the Academy of Sciences of Moldova (project \# 08.820.05.033RF). The author *(E.V.D.) would like to thank the Foundations of young scientists of the Academy of Sciences of Moldova for financial support (project \# 08.819.05.07F).
[1] D. Pines, Elementary Excitations in Solids (Benjamin, New York, 1963).

[2] S. Das Sarma, A. Madhukar, Phys. Rev. B 23, 805 (1981).

[3] A. I. Kasyan, P. I. Rusu, Phys. Status Solidi B 127, 439 (1985).

[4] A. I. Kasyan, I. V. Sur, I. N. Balmush, Fiz. Tekhn. Poluprovodn. 25, 689 (1991).

[5] S. M. Girvin, A. H. MacDonald, P. M. Platzman Phys. Rev. B 33, 2481 (1986).

[6] S. A. Moskalenko, M. A. Liberman, P. I. Khadzhi, E. V. Dumanov, Ig. V. Podlesny, V. Botan, Solid State Commun. 140/5, 236 (2006).

[7] S. A. Moskalenko, M. A. Liberman, P. I. Khadzhi, E. V. Dumanov, Ig. V. Podlesny, V. Botan, Physica E
39, 137 (2007).

[8] L. V. Butov, C. W. Lai, D. S. Chemla, Yu. E. Lozovik, K. L. Campman, A. C. Gossard, Phys. Rev. Lett. 87, 216804 (2001).

[9] N. S. Averkiev, L. E. Golub, A. S. Gurevich, V. P. Evtikhiev, V. P. Kochereshko, A. V. Platonov, A. S. Shkolnik, Y. P. Efimov, Phys. Rev. B 74, 033305 (2006).

[10] S. A. Moscalenko, M. A. Liberman, V. V. Botan, E. V. Dumanov, Ig. V. Podlesny, Mold. J. Phys. Sci. 4, No 2, 21162 (2005).

[11] D. N. Zubarev, Sov. Phys. Usp. Fiz. Nauk 71, 71 (1960).

[12] A. Stefan, in Scientific Conference of Students. Abstracts (CEP USM, Chisinau, 2006), p. 154. 


\title{
ПЛАЗМОВІ КОЛИВАННЯ У ДВОВИМІРНІЙ ЕЛЕКТРОННО-ДІРКОВІЙ РІДИНІ
}

\author{
С. А. Москаленко ${ }^{1}$, М. А. Ліберман ${ }^{2}$, Е. В. Думанов ${ }^{1}$, А. Г. Штефан ${ }^{1}$, М. І. Шмиглюк ${ }^{1}$ \\ ${ }^{1}$ Інститут прикладної фізики Академії наук Молдови, \\ вул. Академічна, 5, Кишинеу, 2028, Республіка Молдова, \\ ${ }^{2}$ Фізичний факультет, Університет Уппсали, Уппсала, Швеція
}

Вивчено плазмові коливання двовимірної електронно-діркової системи у присутності сильного магнітного поля. Враховано лише збудження в межах рівня Ландау, коли електрони і дірки перебувають на відповідних найнижчих рівнях Ландау, а коефіцієнт заповнення $v^{2}<1$. Припускається, що основний стан цієї двовимірної системи є електронно-дірковою рідиною. Отримано дисперсійні співвідношення для оптичних та акустичних плазмонних мод. Проаналізовано вплив додаткового внутрішньоплощинного електричного поля, яке діє поруч із сильним перпендикулярним магнітним полем. 